\title{
Contribution of Radionuclide Theranostics for Managing Intractable Malignancies
}

\author{
Byeong-Cheol Ahn ${ }^{1}$ (D)
}

Received: 30 April 2018 / Revised: 3 May 2018 / Accepted: 4 May 2018 / Published online: 24 May 2018

(C) Korean Society of Nuclear Medicine 2018

\begin{abstract}
Although the survival rate for all cancers is continuing to increase, however there is a substantial percentage of patients that still have not been able to get a long-term survival benefit by not responding to conventional therapies which may incur severe adverse effects and huge medical costs. Heterogeneous biological features of cancers are known to be related to the non-uniform response to the therapies. The heterogeneity of cancer lesions in a single patient is one of the biggest challenges for therapeutic success, and a biopsy of every metastatic lesion is practically impossible in real world clinics [1]. Therefore, pretherapeutic determination of which therapeutic modality would be effective for cancers has significant clinical value by avoiding unnecessary conventional therapeutics and by giving an opportunity for applying other salvage treatments.

Although the term of theranostics was generated after the second millennium, radionuclide theranostics has been successfully employed for oncologic diseases since radioiodine was first used to treat thyroid cancer more than 75 years ago [2-4]. In the era of precision medicine, detecting molecular therapeutic targets in cancers is a major prerequisite for developing the optimal therapeutic strategy for individual patients. Nuclear medicine imaging can non-invasively provide information about the presence of the molecular therapeutic target in individual patients and, furthermore, can provide the information of every lesion in a patient with multiple cancerous lesions, which practically cannot be achieved by conventional invasive histologic analysis [5]. Therefore, radionuclide theranostics can be a promising option for managing intractable malignant diseases.

The radionuclide theranostics, which started in the thyroidologic arena by using free radioiodine, is developing to various oncologic fields by applying sophisticated nuclear,
\end{abstract}

Byeong-Cheol Ahn

abc2000@knu.ac.kr

1 Department of Nuclear Medicine, School of Medicine, Kyungpook National University, Kyungpook National University Hospital, 50, Samduk 2-ga, Jung Gu, Daegu, Republic of Korea 700-721 chemical, biologic, and other technologies [4]. In the territory of radionuclide theranostics, if you are able to see lesions, in general, you can treat the lesions. And you can also predict therapeutic response of each lesion based on what you see. Peptide receptor radionuclide therapy is generated by the marriage between the character of peptides which target certain cancers and the character of radionuclides which emit cytotoxic radiations. At first, the peptide receptor radionuclide therapy was applied for neuroendocrine tumors which express somatostatin receptors and the therapy expanded to other cancers, such as prostate cancers [6]. The beauty of Lu- 177 based peptide receptor radionuclide therapy for neuroendocrine tumors has been discussed in two articles by Kim et al. and by Hirmas et al. [6, 7]. Bone is the most common metastatic site of various cancers, and bone metastasis can result in serious clinical consequences, such as short life expectancy, poor quality of life, etc. For managing bone metastasis, bone targeting radiopharmaceuticals are also effective therapeutic modalities, even though they have their own pros and cons. The principle for and clinical utilities of treating bone metastasis with bone targeting radiopharmaceuticals have been clearly described in one article by Choi [8].

Rapid progress of peptide technology and advances in radiolabeling chemistry will accelerate development of the radionuclide theranostics and also broaden the clinical fields where the therapy works. It is obvious that dedicated clinical researches using theranostic radiopharmaceuticals are urgently required for their successful introduction to routine clinical practice.

Funding This work was supported by the National Research Foundation of Korea (NRF) grant funded by the Korea government (MSIP) (No. NRF2015M2A2A7A01045177).

\section{Compliance with Ethical Standards}

Conflict of Interest Byeong-Cheol Ahn declares no conflict of interest.

Ethical Approval This work does not contain any studies with human participants or animals performed by the author.

Informed Consent under Ethical Approval None. 


\section{References}

1. Junttila MR, de Sauvage FJ. Influence of tumour micro-environment heterogeneity on therapeutic response. Nature. 2013;501:346-54.

2. Jadvar H. Targeted radionuclide therapy: an evolution toward precision cancer treatment. AJR Am J Roentgenol. 2017;209:277-88.

3. Hong CM, Ahn BC. Redifferentiation of radioiodine refractory differentiated thyroid cancer for reapplication of I-131 therapy. Front Endocrinol (Lausanne). 2017;8:260.

4. Ahn BC. Personalized medicine based on theranostic radioiodine molecular imaging for differentiated thyroid cancer. Biomed Res Int. 2016;2016:1680464.
5. Ahn BC. Nuclear medicine in the era of precision medicine. Nucl Med Mol Imaging. 2017;51:99-100.

6. Kim K, Kim S. Lu-177-based peptide receptor radionuclide therapy for advanced neuroendocrine tumors. Nucl Med Mol Imaging. 2018. https://doi.org/10.1007/s13139-017-0505-6.

7. Hirmas N, Jadaan R, Al-Ibraheem A. Peptide receptor radionuclide therapy and the treatment of gastroentero-pancreatic neuroendocrine tumors: current findings and future perspectives. Nucl Med Mol Imaging. 2018. https://doi.org/10.1007/s13139-018-0517-x.

8. Choi JY. Treatment of bone metastasis with bone-targeting radiopharmaceuticals. Nucl Med Mol Imaging. 2018. https://doi.org/10. 1007/s13139-017-0509-2. 